\title{
Evaluation of The Comprehensive Geriatric Assessment (CGA) Tool As A Predictor of Postoperative Complications Following Major Oncological Abdominal Surgery in Older Patients
}

\section{Yoon Penning}

Jules Bordet Institute: Institut Jules Bordet

Antoine El Asmar

Jules Bordet Institute: Institut Jules Bordet

Michel Moreau

Jules Bordet Institute: Institut Jules Bordet

Julie Raspé

Jules Bordet Institute: Institut Jules Bordet

Lissandra Dal Lago

Jules Bordet Institute: Institut Jules Bordet

Thierry Pepersack

Jules Bordet Institute: Institut Jules Bordet

Vincent Donckier

Jules Bordet Institute: Institut Jules Bordet

Gabriel Liberale ( $\nabla$ gabriel.liberale@bordet.be )

Institut Jules Bordet https://orcid.org/0000-0001-8051-1588

\section{Research Article}

Keywords: frailty, geriatric assessment, surgical complication, CGA, G8, intraabdominal

Posted Date: August 13th, 2021

DOI: https://doi.org/10.21203/rs.3.rs-722054/v1

License: (c) (i) This work is licensed under a Creative Commons Attribution 4.0 International License. Read Full License 


\section{Abstract}

Purpose: The concept of frailty extends beyond chronological age. Identifying frailty using a two-step approach, starting with the use of a screening tool (G8) followed by complete geriatric assessment (CGA), may be useful for guiding treatment decisions and follow-up.

This study evaluated the association between geriatric screening and individual components of the tCGA with the risk of 90-day postoperative complications in oncogeriatric patients.

Methods: Data on patients with major oncological abdominal surgery were retrospectively collected from hospital records (2016-2019). Patients with G8 and CGA geriatric screening in those identified by an altered G8 screening score were included. Postoperative complications were classified by Clavien-Dindo grade and the Comprehensive Complication Index (CCl).

Results: One hundred and twelve patients aged $\geq 70$ years surgically treated with curative intent for cancer were included. Seventy-six (67.9\%) presented with an altered G8. Of these, sixty-six $(58.9 \%)$ had a CGA. In univariate analysis, only altered nutritional status assessed by the Mini-Nutritional AssessmentShort Form was associated with higher total complication rate $(p=0.01)$. Patients with impaired G8 had significantly more complications and higher 1-year mortality rates than patients with normal G8. Fifteen patients (13.4\%) had grade III-IVb complications. A CCI > 50 was recorded in 16 patients (14.3\%). Allcause 90 -day postoperative mortality was $10.7 \%$.

Conclusions: Identifying altered preoperative nutritional status as part of the CGA in patients screening positive for frailty is a potentially modifiable risk factor that can influence preoperative management and treatment decision making. G8 may be a predictive factor for postoperative complications.

\section{Introduction}

The age distribution of a population greatly affects its burden of disease and disability, including cancer incidence, morbidity, and mortality [1]. With the sharp increase in life expectancy observed in both men and women, virtually every country in the world is experiencing growth in the size and proportion of older people in their population. Over the next three decades, the global number of older people is projected to more than double, reaching over 1.5 billion by 2050 , with the share of the population aged 65 years or over expected to reach 16 percent [2].

Although the relationship between ageing and cancer is complex and far from understood, the incidence of cancer increases with age in humans and in laboratory animals alike [3]. As global epidemiologic and demographic transitions continue, they signal an ever-growing cancer burden over the next few decades, with over 20 million new cases expected annually as early as 2025 . With surgical intervention continuing to be the principal treatment for many solid tumors, the number of older patients undergoing surgery as part of their cancer treatment is also expected to rise [4]. 
These older patients are often considered at increased risk for complications after major surgery but chronological age alone is not a reliable predictor of postoperative complications [5]. As an isolated factor, it is insufficient to capture the physiologic heterogeneity prevalent in this population [6]. The concept of frailty extends beyond chronological age and is one of the most serious global health challenges to be faced in the coming century. Frailty can be defined as "a complex clinical condition characterized by a decline in physiological capacity and reserve across several organ systems, with a resultant increased susceptibility to stressors" [7]. Older patients considered fit for surgery might do as well as younger patients but frail and vulnerable patients are at an increased risk of adverse postoperative outcomes [8]. The usual method used to identify frailty is to evaluate an older patient's general condition and risk of adverse outcomes using the evidence-based process of comprehensive geriatric assessment (CGA). Through a battery of standardized and validated assessment instruments, CGA evaluates nutrition, cognition, functional status, comorbidities, and geriatric syndromes to identify at-risk patients and to possibly guide management, treatment, and follow-up [9].

Since administering a complete geriatric assessment is time-consuming, the International Society of Geriatric Oncology (SIOG) recommends a "two-step approach". This strategy starts with the use of a screening tool to identify patients in need of further evaluation by CGA. Screening can be done with the Geriatric 8 (G8), a screening tool that includes seven items from the Mini Nutritional Assessment (MNA) and an age-related item $(<80,80-85$, or $>85$ years). Final scores range from 0 to 17 , with a score below 14 indicating a geriatric risk profile [10]. Some components of the CGA have been found to be consistently associated with adverse postoperative outcomes; however, based on current evidence, it is not possible to reach a consensus as to how an optimal geriatric assessment (GA) should be conducted and recommendations vary based on patient age, type of surgery (i.e., minor versus major), and other risk factors $[8,11]$. Moreover, geriatric screening using the $\mathrm{G} 8$ assessment tool has been reported to be a powerful outcome predictor in surgical oncogeriatric patients in terms of hospital stay, rate of postoperative delirium, and 1-year mortality rates [12].

The purpose of this study was to identify independent predictors of postoperative complications in older surgical patients undergoing major oncological abdominal surgery based on components of the comprehensive geriatric assessment. The secondary aim of our study was to investigate differences in postoperative outcomes between patients identified as frail and those with a normal G8 score.

\section{Materials And Methods}

\section{Study design \& objectives}

This was a monocentric retrospective study including patients $\geq 70$ years old treated for abdominal cancer with surgery with a curative intent at Institut J. Bordet (IJB) between January 2016 and December 2019. The study protocol was reviewed and approved by the Ethics Committee of the IJB (CE3277). For the retrospective review of electronic medical records, written informed consent was not needed. 
The primary objective of the study was to identify whether some components of the CGA are independent predictors of postoperative complications in oncogeriatric patients.

The secondary objective was to evaluate the predictive value of G8 in postoperative complications between patients identified as frail due to an altered G8 score and those with a normal G8 score.

\section{Data sources}

We retrospectively researched the hospital's records of all surgical interventions performed during the study period. Major surgical interventions were defined based on the extent of dissection: major body cavity opened (e.g. peritoneal cavity) and as oncological surgeries with major-to-severe tissue trauma (e.g. resection of an organ or part of it and/or digestive anastomosis). Inclusion criteria included patients 70 years or older, scheduled for major oncological abdominal surgery, such as: hepatectomy (open or laparoscopic), colectomy (open or laparoscopic), abdomino-perineal resection, cytoreductive surgery with or without hyperthermic intraperitoneal chemotherapy (HIPEC), rectal resection, small bowel resection, exploratory laparotomy, esophagectomy, and gastrectomy. Further inclusion criteria among those patients were: pre-operative geriatric 8 (G8) screening as well as a comprehensive geriatric assessment in those scoring below or equal to 14 when evaluated by the $\mathrm{G} 8$ screening tool, performed at least 3 months prior to surgical intervention. All other patients were excluded (e.g. emergency surgeries).

The institutional electronic medical record system (Oribase) was used as the data source. Among different components of the CGA, we retained a set of seven validated scores and questionnaires:

- Katz's activities of daily living (ADL) and Lawton's instrumental activities of daily living (IADL) scales to assess functional status $[13,14]$

- Mini-Mental State Examination (MMSE) to assess cognitive status [15]

- Geriatric Depression Scale (GDS) to assess depression status [16]

- Mini-Nutritional Assessment Short Form (MNA-SF) to assess nutrition status [17]

- Hospital Anxiety and Depression Scale (HADS-A HADS-D) to assess the symptom severity of anxiety disorders and depression [18]

Patients with missing values on one or more instruments were also included.

Postoperative complications obtained from medical records were defined as any event requiring treatment occurring in a 90-day period after the intervention. Severity of complications were classified by the primary investigator according to the Clavien-Dindo classification scale (grades I to V) [19]. Grades I and II were considered minor complications whereas grades IIIa to IVb were recorded as major complications. Grade $\mathrm{V}$ meant death of the patient. For complications requiring more than one treatment method, the highest severity grade was noted. In case of multiple complications in a patient, each was recorded and graded separately and, additionally, the Comprehensive Complication Index (CCI) was calculated and reported on an interval scale from 0 to 100 to summarize all (minor and major) complications [20]. Postoperative mortality was defined as death within 90 days. Data for age, gender, 
cancer type, histology, type of intervention, CGA results, and complications were obtained from the institutional medical records system (Oribase).

\section{Statistical Methods}

Descriptive analysis of clinical and demographic variables was performed. All categorical variables are described using numbers $(\mathrm{N})$ and percentages $(\%)$ whereas continuous variables are described using means.

\section{Geriatric Assessment}

In the subgroup of patients scoring below or equal to 14 at G8 survey and having received a complete geriatric assessment, patients were divided into two groups based on median values of continuous variables for each score and questionnaire. Dichotomized outcome variables for 90 -day postoperative complications were created according to Clavien-Dindo: no complications versus any complications. The impact of the collected variable (assessment tool of the comprehensive geriatric assessment) upon the variable of dependent interest (postoperative complications) was explored by univariate analysis and by fitting logistic regression models using the six subscales of the geriatric assessment.

\section{G8 screening tool}

Development of complications was compared between patients with a G8 $\leq 14$ and a G8 $>14$. This study investigated postoperative outcomes using binary measures: 1 . Any morbidity (grade I-V) within 90 days of surgery, 2. Major morbidity (grade $>\mathrm{II}$ ) within 90 days of surgery, 3. Major $\mathrm{CCl}$ score $(\mathrm{CCl}>50) 4$. Death (grade $\mathrm{V}$ ) within 90 days of surgery.

To test the significance of each variable in relation to the outcomes, univariate analyses were performed using the chi-square test or Fisher's exact test. Statistical information was encoded anonymously into a database using Microsoft Excel spreadsheets. A value of $p<0.05$ was considered to be statistically significant. All analyses were done using the Statistical Package for the Social Sciences (SPSS, version 27.0, New York, US).

\section{Results}

\section{Baseline patient characteristics}

A total of 1377 patients underwent an elective abdominal surgery in the inclusion period. Of these, 1156 patients were excluded as they had minor surgeries. This included non-oncological surgeries (e.g. cholecystectomy, umbilical or inguinal hernias) and oncological surgeries with a minimal or low amount of tissue trauma and limited extent of dissection (e.g. digestive stoma). A total of 571 were excluded because the patients were younger than 70 years of age at the time of surgery. 
From the 221 interventions for oncological abdominal surgery remaining, 69 (31.2\%) were further excluded because no G8 screening was performed. Of the remaining $152,40(26.3 \%)$ were excluded for not having an appropriate G8 performed; screening was either performed after the intervention or more than 3 months prior. An overview of the flow of patient inclusion into the study is provided in Figure 1.

Among the 112 patients we retained for analysis, the median age was 74 years and 46 were male (41.1\%). The two most common tumor sites were colorectal $(59,52.7 \%)$ and ovarian $(15,13.4 \%)$ and the most common histology type was adenocarcinoma $(95,84.8 \%)$. The majority of surgeries $(82,71.4 \%)$ were open surgeries and the most frequent types of intervention were colectomy $(29,25.9 \%)$ and cytoreductive surgery $(20,17.9 \%)$. Malignancies classified as "other" included unknown primaries, endometrial tumors, biliary tract cancers, pancreato-biliary cancers, retroperitoneal cancers and breast, lung, and skin cancers metastatic to the abdomen. A majority of patient cancers $(42,37.5 \%)$ were classified as grade II. Details of baseline clinical variables are summarized in Table 1. G8 was altered in 76 patients $(67.9 \%)$ and normal in 36 patients $(32.1 \%)$. When we compared the characteristics of the two groups according to sex, cancer type, tumor histology, type of intervention, and surgical approach, we did not find significant differences except for cancer grade ( $p$ value 0.04 ).

\section{Comprehensive Geriatric Assessment}

Among the 76 patients with an altered G8, the median age was 76 years ( $70-92$ years). Ten of these patients were not evaluated by CGA at all. Preoperative CGA scores among the remaining 66 patients were $\mathrm{ADL}>6$ in $33.3 \%$, IADL $\leq 7$ in $50.8 \%$, MMSE $\leq 28$ in $57.9 \%$, GDS > 3 in $50.0 \%$, HADS-A > 6 in $42.9 \%$, HADS-D $>4$ in $46.4 \%$, and MNA-SF $\leq 9$ in $39.4 \%$.

\section{Postoperative outcomes}

Out of all included patients ( $\mathrm{N}=112), 77$ developed at least one complication. The majority of patients $(43,38.4 \%)$ experienced a grade II complication and in 15 patients $(13.4 \%)$ a major complication was reported. Sixteen patients $(14.3 \%)$ had a $\mathrm{CCl}$ score $>50$. Respectively, twelve patients $(10.7 \%)$ died within the 90-day follow-up period from all causes and from postoperative complications. Postoperative Clavien-Dindo scores are displayed in Table 2.

\section{Association between CGA and complications}

We analyzed the impact of each component of the GA upon the development of complications. In univariate analysis, the short form of the Mini-Nutritional Assessment was the sole prognostic factor of complications $(p=0.03)$. There were no associations between the other assessment tools analyzed and complications. Results are shown in Table 3. 


\section{Association between $\mathbf{G 8}$ and complications}

Table 4 provides an overview of the univariate analysis for predictors of complications, higher CD-score, higher $\mathrm{CCl}$ score, and mortality. A G8 score below or equal to 14 was significantly associated with the development of complications within 90 days of surgery. Patients who screened positive for frailty had significantly more complications than patients who screened negative, $52 \%$ versus $17 \%(p<0.05)$, respectively. However, altered G8 was not associated with the occurrence of major complications or higher $\mathrm{CCl}$ score. Additionally, no association was reported between altered $\mathrm{G} 8$ and 90 -day mortality but it was, however, significantly associated with a higher 1 -year mortality ( $p$ value 0.01 ).

\section{Discussion}

The primary aim of our study was the identification of independent predictors of postoperative complications based on components of the CGA. In our population, $67.9 \%$ of the patients were identified by an altered $\mathrm{G} 8$ as being at risk for frailty. Among those patients, 26 scored below or equal to 9 when evaluated by the short form of the Mini-Nutritional Assessment for nutritional status. Our main finding was the strong association between this nutrition-based evaluation tool and the occurrence of any complications during the 90-day postoperative course. In this group of patients with an abnormal G8 score, no association was found between other components of the CGA and postoperative complications. This relationship has been increasingly investigated in the field of surgical oncology but with inconsistent results. In patients with colorectal cancer, ADL, IADL, MMSE, GDS, and MNA were inconsistently associated with postoperative complications [9]. Huisman et al. found impaired nutrition to be associated with major postoperative complications but nutrition status was evaluated by Nutritional Risk Screening (NRS) in that study [8]. For patients undergoing surgery for gastric cancer resection, other malnutrition screening tools have been validated and found to be associated with peri-operative and postoperative morbidity and complications [21].

Our findings indicate the importance of preoperative nutritional assessment in major oncological abdominal surgery in order to provide valuable patient information, to assess surgical risk-benefit ratio, and to possibly provide individualized nutritional therapy by multidisciplinary teams even though the impact of normalization of potentially reversible factors on postoperative complications is still under investigation [22]. However, despite recommendations on geriatric assessment in older patients with cancer published in 2005 by the SIOG, there is no consensus regarding which specific instruments should be included in a CGA [12]. Inclusion of nutritional impairment screening is of high interest as this reversible factor seems to be an indicator of increased postoperative morbidity. Heterogeneity among patient selection and surgical intervention makes comparison difficult and indicates the need for further studies with larger populations using identical screening tools and cut-off values.

Another important finding in our study was the significant difference in postoperative outcomes between patients with a G8 $\leq 14$ and patients with a G8 $>14$. We identified an association between altered G8 screening and the development of at least one complication. Several studies investigating this 
relationship have provided contradictory results. A recent study on preoperative frailty assessment in 114 patients found the G8 tool to not be significantly associated with the risk of adverse events [23]. Another study found similar results in 139 older patients treated surgically for colorectal cancer. The authors did not find isolated G8 to be of any predictive value on postoperative outcomes. They did, however, find that a combination of the G8 and the Identification of Seniors at Risk-Hospitalized Patients (ISAR-HP) screening tool resulted in a high predictive value [24]. Another combination was recently investigated. Bessems et al. indicated that frailty screening by G8 in association with the 4-meter gait speed test predicts postoperative complications in colorectal cancer patients undergoing elective surgery [25]. Additionally, in another study, de Vries et al. found G8 to be a strong predictor of postoperative complications in a population of patients undergoing surgery for cutaneous head and neck cancer [26].

Our study did not find a relation between altered G8 and major complications (defined as Clavien-Dindo grade $>$ II). When investigating this same relationship in 143 patients of the same age group, requiring surgery for a suspected solid malignancy, Bruijnen et al., found no difference in the occurrence of major 30-day complications [27]. However, three studies did describe an association between G8 and the occurrence of major Clavien-Dindo complications. One study included grade II complications and found this association in 78 patients who underwent surgery for colorectal cancer [9], while another in 184 patients who underwent emergency abdominal surgery (including non-oncological patients) [28] and a third in 71 patients treated for hepatocellular carcinoma [12] confirmed these findings.

Furthermore, in these studies, no association was found between altered G8 and a higher 1-year mortality rate. We found altered $\mathrm{G} 8$ to not be predictive of 90-day mortality in our population. It was, however, associated with increased 1-year mortality. This may be explained by significantly higher cancer grades in this group.

The predictive value of the $\mathrm{G} 8$ on postoperative complications in surgical oncology and practice remains unclear and further trials of larger populations are needed to complete our understanding of this screening tool and its place in diagnostic algorithms. This is in contrast to studies done in non-surgical oncological patients, in whom altered G8 has been shown to provide helpful information through prediction of complications in patients receiving chemotherapy and/or radiotherapy [29]. Furthermore, it is imperative to mention that the G8 was initially developed as a frailty screening tool for predicting deficits in the CGA. It was not intended to be used as a prognostic tool.

Direct comparison between these study results and our results should be approached with caution. The above-mentioned studies focused mostly on homogenous populations with a single malignancy or surgical intervention whereas our study included a variety of tumor types and surgeries. Heterogeneity remains among patient characteristics between studies, definitions of frailty, cut-off values for screening and assessment tools but also in the definition of adverse postoperative outcomes.

The present study had several limitations. First, although the inclusion criteria limited our population to patients undergoing 'major' oncological abdominal surgery, heterogeneity affecting risk of complications remained. For example, Kothari et al. recently showed that within each of their frailty cohorts, 
laparoscopic colectomy provided better outcomes when compared to an open approach in all domains, including cardiac/vascular, pulmonary, and wound complications [30]. Additionally, this study did not account for differences in tumor characteristics, such as stage, treatment course, or intensity. Another limitation is the monocentric nature of our study since variations in postoperative complications are influenced by technical skill scores of surgeons and quality of care by medical staff [31,32]. Other limitations lie in the fact that postoperative outcomes were collected retrospectively. As a result, some minor complications may have been underreported. Moreover, $31 \%$ of patients $\geq 70$ years were not evaluated because they did not undergo G8 screening and this could have created bias in the analyses. Despite these limitations, the study also had some strengths. Our study was unique as our study population was composed of only older patients admitted for major oncological abdominal surgery not centered around a single malignancy or surgical approach.

In conclusion, this study suggests that the MNA-SF is a valuable asset in preoperative risk assessment for complications that have the potential to impede recovery and its use could be beneficial in the everyday practice of surgical abdominal oncology. Evaluation of nutritional status as part of the comprehensive geriatric assessment in patients identified as frail seems essential as nutrition is a potentially modifiable factor influencing preoperative management and treatment modalities. G8, besides its role as a screening tool for impairment in the CGA, shows potential as a predictor of postoperative complications. This finding corroborates well with the MNA's predictive potential since all the questions of the G8 - except for age - are derived from the MNA.

\section{Declarations}

Acknowledgements: The authors acknowledge the contribution of a medical writer, Sandy Field, PhD, for English language editing and formatting of this manuscript.

Funding: The authors received no funding for this study.

Conflicts of interest/Competing interest: The authors have no conflicts of interest to declare.

Availability of data and material: at request to authors

Code availability: NA

Author's contributions: Gabriel Liberale designed the study. Yoon Penning \& Julie Raspé collected the data. Yoon Penning wrote the manuscript. Lissandra Dal Lago and Thierry Pepersack have participated to the G8 and GA evaluation (prospectively), Antoine El Asmar \& Michel Moreau performed the data analysis. Donckier, Gabriel Liberale, Lissandra Dal Lago and Thierry Pepersack reviewed and edited the manuscript.

Ethics approval: The study protocol was reviewed and approved by the Ethics Committee of the IJB (CE3277). 
Consent to participate: For the retrospective review of electronic medical records, written informed consent was not needed.

Consent for publication: For the retrospective review of electronic medical records, written informed consent was not needed.

\section{References}

1. Repetto L, Venturino A, Fratino L, Serraino D, Troisi G, Gianni W, Pietropaolo M (2003) Geriatric oncology: a clinical approach to the older patient with cancer. Eur J Cancer 39:870-880. https://doi.org/10.1016/s0959-8049(03)00062-5

2. United Nations, Department of Economic and Social Affairs, Population Division (2020) World population ageing, 2019 highlights

3. Anisimov VN, Sikora E, Pawelec G (2009) Relationships between cancer and aging: a multilevel approach. Biogerontology 10:323-338. https://doi.org/10.1007/s10522-008-9209-8

4. Plas $M$, de Haan JJ, van der Wal-Huisman $H$, Rutgers $A$, Absalom AR, de Bock $G H$, van Leeuwen $B L$ (2019) The systemic impact of a surgical procedure in older oncological patients. Eur J Surg Oncol 45:1403-1409. https://doi.org/10.1016/j.ejso.2019.04.024

5. Badgwell B, Stanley J, Chang GJ, Katz MHG, Lin HY, Ning J, Klimberg SV, Cormier JN (2013) Comprehensive geriatric assessment of risk factors associated with adverse outcomes and resource utilization in cancer patients undergoing abdominal surgery. J Surg Oncol 108:182-186. https://doi.org/10.1002/jso.23369

6. Richards SJG, Frizelle FA, Geddes JA, Eglinton TW, Hampton MB (2018) Frailty in surgical patients. Int J Colorectal Dis 33:1657-1666. https://doi.org/10.1007/s00384-018-3163-y

7. Dent E, Martin FC, Bergman H, Woo J, Romero-Ortuno R, Walston JD (2019) Management of frailty: opportunities, challenges, and future directions. Lancet 394:1376-1386.

https://doi.org/10.1016/s0140-6736(19)31785-4

8. Huisman MG, Kok M, de Bock GH, van Leeuwen BL (2017) Delivering tailored surgery to older cancer patients: Preoperative geriatric assessment domains and screening tools - A systematic review of systematic reviews. Eur J Surg Oncol 43:1-14

9. Fagard K, Casaer J, Wolthuis A, Flamaing J, Milisen K, Lobelle J-P, Wildiers H, Kenis C (2017) Value of geriatric screening and assessment in predicting postoperative complications in patients older than 70 years undergoing surgery for colorectal cancer. J Geriatr Oncol 8:320-327. https://doi.org/10.1016/j.jgo.2017.07.008

10. Kaibori M, Ishizaki M, Matsui K, lida H, Inoue K, Nagashima F, Kon M (2016) Geriatric assessment as a predictor of postoperative complications in elderly patients with hepatocellular carcinoma. Langenbecks Arch Surg 401:205-214. https://doi.org/10.1007/s00423-016-1388-1

11. Feng MA, McMillan DT, Crowell K, Muss H, Nielsen ME, Smith AB (2015) Geriatric assessment in surgical oncology: A systematic review. J Surg Res 193:265-272. 
https://doi.org/10.1016/j.jss.2014.07.004

12. Bruijnen CP, Heijmer A, van Harten-Krouwel DG, van den Bos F, de Bree R, Witteveen PO, EmmelotVonk MH (2021) Validation of the G8 screening tool in older patients with cancer considered for surgical treatment. J Geriatr Oncol 12:793-798. https://doi.org/10.1016/j.jgo.2020.10.017

13. Katz S, Ford AB, Moskowitz RW, Jackson BA, Jaffe MW (1963) Studies of Illness in the Aged: The Index of ADL: A Standardized Measure of Biological and Psychosocial Function. JAMA 185:914919. https://doi.org/10.1001/jama.1963.03060120024016

14. Lawton MP, Brody EM (1969) Assessment of older people: self-maintaining and instrumental activities of daily living. Gerontologist 9:179-186

15. Folstein MF, Folstein SE, McHugh PR (1975) “Mini-mental state”. A practical method for grading the cognitive state of patients for the clinician. J Psychiatr Res 12:189-198. https://doi.org/10.1016/0022-3956(75)90026-6

16. Yesavage JA, Brink TL, Rose TL, Lum O, Huang V, Adey M, Leirer VO (1982-1983) Development and validation of a geriatric depression screening scale: a preliminary report. J Psychiatr Res 17:37-49. https://doi.org/10.1016/0022-3956(82)90033-4

17. Kaiser MJ, Bauer JM, Ramsch C, Uter W, Guigoz Y, Cederholm T, Thomas DR, Anthony P, Charlton KE, Maggio M, Tsai AC, Grathwohl D, Vellas B, Sieber CC, MNA-International Group (2009) Validation of the Mini Nutritional Assessment short-form (MNA-SF): a practical tool for identification of nutritional status. J Nutr Health Aging 13:782-788. https://doi.org/10.1007/s12603-009-0214-7

18. Zigmond AS, Snaith RP (1983) The hospital anxiety and depression scale. Acta Psychiatr Scand 67:361-370. https://doi.org/10.1111/j.1600-0447.1983.tb09716.x

19. Clavien PA, Barkun J, de Oliveira ML, Vauthey JN, Dindo D, Schulick RD, de Santibañes E, Pekolj J, Slankamenac K, Bassi C, Graf R, Vonlanthen R, Padbury R, Cameron JL, Makuuchi M (2009) The Clavien-Dindo classification of surgical complications: five-year experience. Ann Surg 250:187-196. https://doi.org/10.1097/sla.0b013e3181b13ca2

20. Ray S, Mehta NN, Mangla V, Lalwani S, Mehrotra S, Chugh P, Yadav A, Nundy S (2019) A Comparison Between the Comprehensive Complication Index and the Clavien-Dindo Grading as a Measure of Postoperative Outcome in Patients Undergoing Gastrointestinal Surgery-A Prospective Study. J Surg Res 244:417-424. https://doi.org/10.1016/j.jss.2019.06.093

21. Kanda M (2020) Preoperative predictors of postoperative complications after gastric cancer resection. Surg Today 50:3-11. https://doi.org/10.1007/s00595-019-01877-8

22. Lee DU, Fan GH, Hastie DJ, Addonizio EA, Suh J, Prakasam VN, Karagozian R (2021) The clinical impact of malnutrition on the postoperative outcomes of patients undergoing colorectal resection surgery for colon or rectal cancer: Propensity score matched analysis of 2011-2017 US hospitals. Surg Oncol 38:101587. https://doi.org/10.1016/j.suronc.2021.101587

23. Nishijima TF, Esaki T, Morita M, Toh Y (2021) Preoperative frailty assessment with the Robinson Frailty Score, Edmonton Frail Scale, and G8 and adverse postoperative outcomes in older surgical patients with cancer. Eur J Surg Oncol 47:896-901. https://doi.org/10.1016/j.ejso.2020.09.031 
24. Souwer ETD, Verweij NM, van den Bos F, Bastiaannet E, Slangen RME, Steup WH, Hamaker ME, Portielje JEA (2018) Risk stratification for surgical outcomes in older colorectal cancer patients using ISAR-HP and G8 screening tools. J Geriatr Oncol 9:110-114. https://doi.org/10.1016/j.jgo.2017.09.003

25. Bessems SAM, Konsten JLM, Vogelaar JFJ, Csepán-Magyar R, Maas HAAM, van de Wouw YAJ et al. Frailty screening by Geriatric-8 and 4-meter gait speed test is feasible and predicts postoperative complications in elderly colorectal cancer patients. J Geriatr Oncol [Internet]. 2020 Nov 4 [cited 2021 May 1]; Available from: https://www.sciencedirect.com/science/article/pii/S1879406820304847

26. de Vries J, Heirman AN, Bras L, Plaat BEC, Rácz E, van Kester MS, Festen S, de Bock GH, van der Laan BFAM, Halmos GB (2020) Geriatric assessment of patients treated for cutaneous head and neck malignancies in a tertiary referral center: Predictors of postoperative complications. Eur J Surg Oncol 46:123-130. https://doi.org/10.1016/j.ejso.2019.08.008

27. Bruijnen CP. Validation of the G8 screening tool in older patients with cancer considered for surgical treatment. 2020;6

28. Kenig J, Zychiewicz B, Olszewska U, Barczynski M, Nowak W (2015 Dec) Six screening instruments for frailty in older patients qualified for emergency abdominal surgery. Arch Gerontol Geriatr 61(3):437-442

29. van Walree IC, Scheepers E, van Huis-Tanja L, Emmelot-Vonk MH, Bellera C, Soubeyran P et al (2019) A systematic review on the association of the $\mathrm{G} 8$ with geriatric assessment, prognosis and course of treatment in older patients with cancer. J Geriatr Oncol 10(6):847-858

30. Kothari P, Congiusta DV, Merchant AM (2019) Laparoscopic versus open colectomy: the impact of frailty on outcomes. Updates Surg 71:89-96. https://doi.org/10.1007/s13304-018-0531-7

31. Stulberg JJ, Huang R, Kreutzer L, Ban K, Champagne BJ, Steele SR, Johnson JK, Holl JL, Greenberg CC, Bilimoria KY (2020) Association Between Surgeon Technical Skills and Patient Outcomes. JAMA Surg 155:960-968. https://doi.org/10.1001/jamasurg.2020.3007

32. Mokutani Y, Mizushima T, Yamasaki M, Rakugi H, Doki Y, Mori M (2016) Prediction of Postoperative Complications Following Elective Surgery in Elderly Patients with Colorectal Cancer Using the Comprehensive Geriatric Assessment. Dig Surg 33:470-477. https://doi.org/10.1159/000446709

\section{Tables}


Table 1

Clinical and Demographic Variables

\begin{tabular}{|c|c|c|c|}
\hline Variable & $\mathbf{N}$ & $\%$ & $p$ value \\
\hline Age-median (Range) & \multicolumn{3}{|c|}{74 years $(70-92)$} \\
\hline Gender & 66 & 58.9 & 0.62 \\
\hline \multicolumn{4}{|l|}{ Female } \\
\hline Male & 46 & 41.1 & \\
\hline Cancer Type & 59 & 52.7 & 0.88 \\
\hline \multicolumn{4}{|l|}{ Colorectal } \\
\hline Ovary & 15 & 13.4 & \\
\hline Liver & 6 & 5.3 & \\
\hline Stomach & 9 & 8.0 & \\
\hline Small Bowel & 2 & 1.8 & \\
\hline Esophagus & 5 & 4.46 & \\
\hline Other & 16 & 14.29 & \\
\hline Histology Adenocarcinoma & 95 & 84.8 & 0.59 \\
\hline Hepatocarcinoma & 5 & 4.7 & \\
\hline Soft tissue sarcoma & 2 & 1.8 & \\
\hline Cholangiocarcinoma & 2 & 2.8 & \\
\hline Epidermoid Carcinoma & 3 & 2.7 & \\
\hline Other & 5 & 4.5 & \\
\hline Type of intervention & 29 & 25.9 & 0.53 \\
\hline \multicolumn{4}{|l|}{ Colectomy (open or laparoscopic) } \\
\hline Cytoreductive Surgery & 20 & 17.9 & \\
\hline Hepatectomy (open or laparoscopic) & 16 & 14.3 & \\
\hline Anterior Resection of Rectum & 12 & 10.7 & \\
\hline Exploratory Laparotomy & 10 & 8.9 & \\
\hline Cytoreductive Surgery with HIPEC & 5 & 4.5 & \\
\hline Abdominoperineal Amputation & 8 & 7.1 & \\
\hline Gastrectomy & 6 & 5.3 & \\
\hline
\end{tabular}




\begin{tabular}{|llll|}
\hline Variable & N & \% & p value \\
\hline Esophagectomy (total or subtotal) & 3 & 2.7 & \\
Small Bowel Resection & 3 & 2.7 & \\
Cancer grade & 29 & 25.9 & $\mathbf{0 . 0 4}$ \\
I & & & \\
II & 42 & 37.5 & \\
III & 28 & 25.0 & \\
\hline Unknown & 13 & 11.5 & \\
\hline Type of surgery & & & 0.2 \\
\hline Open & 82 & 71.4 & \\
\hline Laparoscopic & 32 & 28.6 & \\
\hline
\end{tabular}

Table 2

Postoperative Clavien-Dindo scores

\begin{tabular}{|lcccccccc|}
\hline & $\mathbf{0}$ & I & II & Illa & IIIb & IVa & IVb & V \\
\hline $\mathbf{G} \leq \mathbf{1 4}(\mathbf{N}=\mathbf{7 6})$ & 18 & 5 & 33 & 6 & 3 & 1 & 0 & 10 \\
\hline $\mathbf{G 8}>\mathbf{1 4}(\mathbf{N}=\mathbf{3 6})$ & 17 & 2 & 10 & 3 & 2 & 0 & 0 & 2 \\
\hline Total & 35 & 7 & 43 & 9 & 5 & 1 & 0 & 12 \\
\hline
\end{tabular}


Table 3

Univariate Analysis for Predictors of Postoperative Complications

\begin{tabular}{|lllll|}
\hline & \multicolumn{3}{ll}{ Complications } & \\
\cline { 3 - 5 } & & Yes & No & p value \\
\hline Score & Total $(\mathrm{N}-\%)$ & $\mathrm{N}-\%$ & $\mathrm{~N}-\%$ & \\
\hline ADL & & & & 0.35 \\
\hline$\leq 6$ & $44-66.7 \%$ & $33-75 \%$ & $11-25 \%$ & \\
\hline$>6$ & $22-33.3 \%$ & $19-86.4 \%$ & $3-13.6 \%$ & \\
\hline IADL & & & & 0.44 \\
\hline$\leq 7$ & $33-50.8 \%$ & $27-81.8 \%$ & $6-18.2 \%$ & \\
\hline$>7$ & $32-49.2 \%$ & $24-73.5 \%$ & $8-24.2 \%$ & \\
\hline MMSE & & & & 0.20 \\
\hline$\leq 28$ & $33-57.9 \%$ & $28-84.8 \%$ & $5-15.2 \%$ & \\
\hline$>28$ & $24-17.1 \%$ & $17-70.8 \%$ & $7-29.2 \%$ & \\
\hline GDS & & & & 0.48 \\
\hline$\leq 3$ & $36-59.0 \%$ & $30-83.3 \%$ & $6-16.7 \%$ & \\
\hline$>3$ & $25-50.0 \%$ & $19-76.0 \%$ & $6-24.0 \%$ & \\
\hline HADS-A & & & & 0.57 \\
\hline$\leq 6$ & $32-57.1 \%$ & $26-81.3 \%$ & $6-18.8 \%$ & \\
\hline$>6$ & $24-42.9 \%$ & $18-75.0 \%$ & $6-25.0 \%$ & \\
\hline HADS-D & & & & \\
\hline$\leq 4$ & $30-53.6 \%$ & $25-83.3 \%$ & $5-16.7 \%$ & \\
\hline MNA-SF & & & & \\
\hline$\leq 9$ & $26-39.4 \%$ & $24-92.31 \%$ & $2-7.7 \%$ & \\
\hline$>9$ & $40-60.6 \%$ & $28-70 \%$ & $12-30 \%$ & \\
\hline
\end{tabular}


Table 4

Univariate Analysis for Predictors of Complications, Higher CD-Score, Higher CCI Score, 90-Day and 1-year mortality

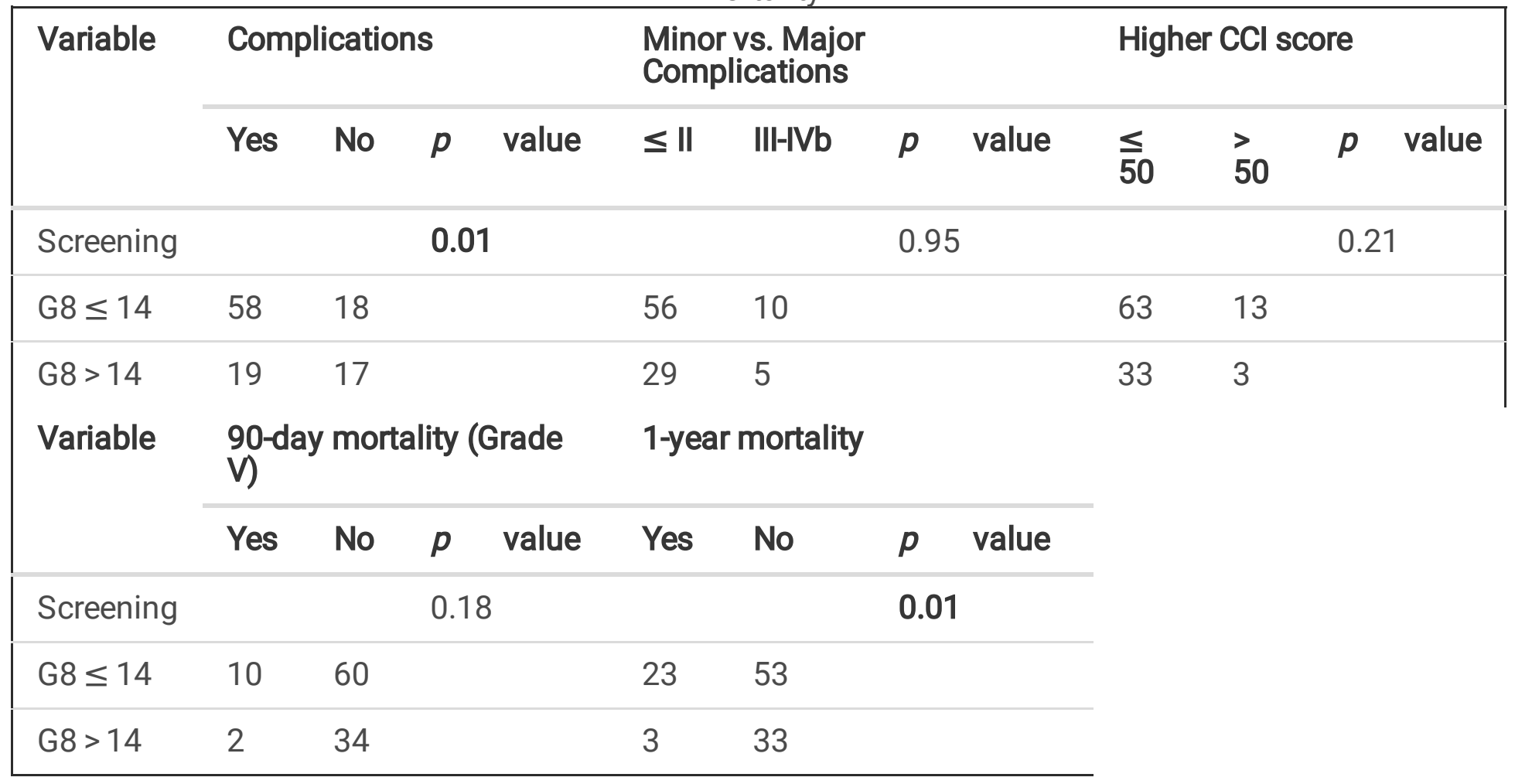

Figures 


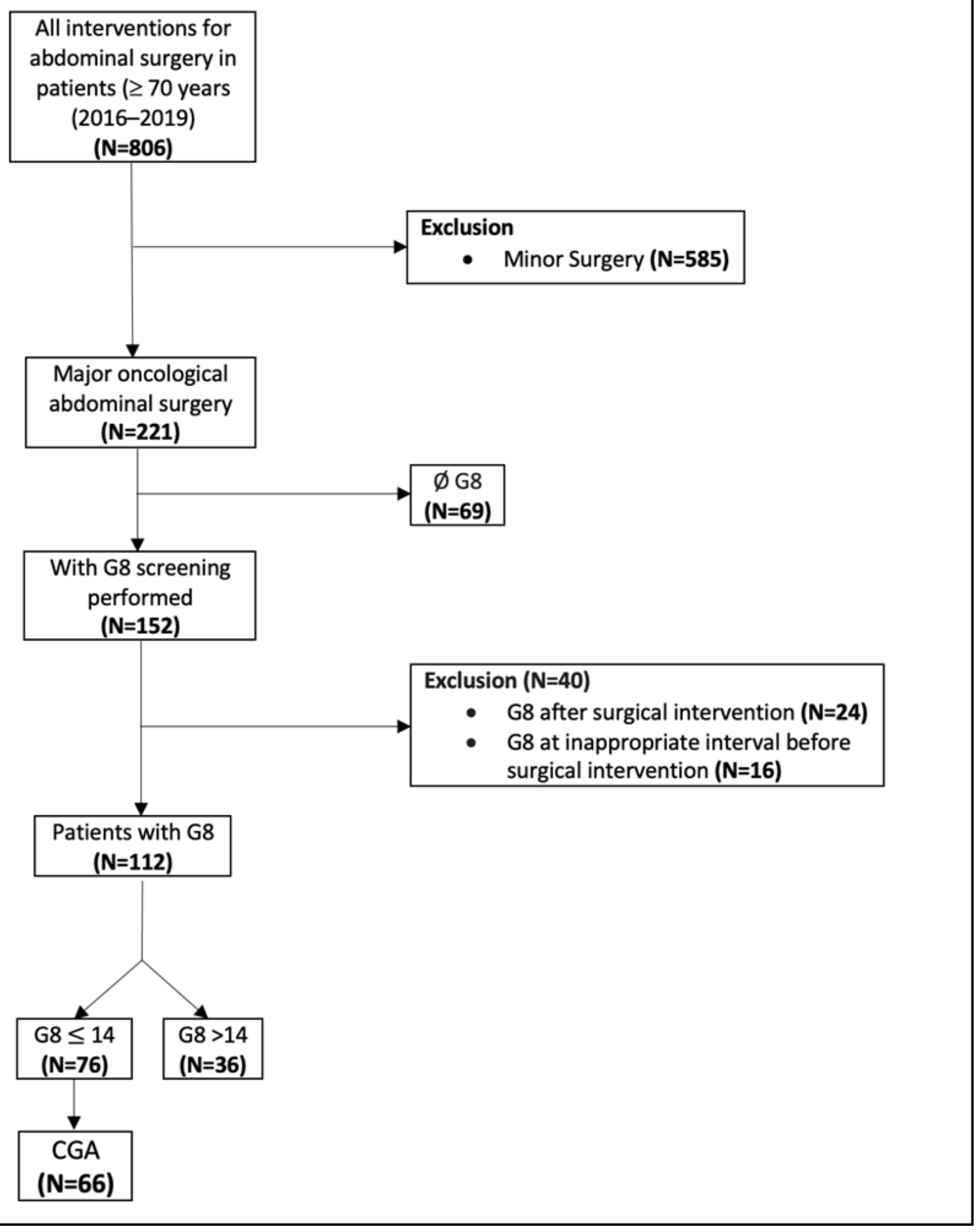

Figure 1

Flowchart of patient inclusion in the study 